\title{
Towards A Personalised Rehabilitation System for Post Stroke Treatment
}

\author{
Nick R. Harris, Dinesh Sthapit \\ Electronics and Computer Science \\ University of Southampton \\ Southampton, United Kingdom
}

\begin{abstract}
Rehabilitation of post-stroke patients involves long-term care from a large team of professionals and constitutes a major portion of the cost of illness for stroke. Assistive technology that allows patients to perform rehabilitation routines themselves, could minimize costly human intervention and help reduce the impact of stroke on the economy and healthcare system. Due to the limited motor ability of post-stroke patients, rehabilitation technology aimed at stroke patients, must focus on ease of use as well as maintaining interest in the exercise. To this end, this paper presents the design of a system for post-stroke rehabilitation, which addresses both these problems by including novel forms of feedback (light and sound) to encourage and maintain progress, and provide reduced set-up time to emphasise ease of use by using a con-contact distance measuring system. The MGC3130 gesture and tracking controller was used to analyse finger motion and thus allowing the calculation maximum finger extension, speed of finger movement and jerks or wobbles in motion per second from a simple training session consisting of up-down finger motion with fixed number of repetitions for each finger. This paper focusses on this sensor subsystem and reports that finger extension can be calculated with an accuracy of about two millimetres within an extension range of two centimetres, allowing future work to investigate the performance of the whole system. Keywords-distance, capacitive, sensor, rehabilitation, stroke
\end{abstract}

\section{INTRODUCTION}

Stroke is a global health issue and is the second most common cause of death and disability worldwide. It occurs as a result of sudden interruption in blood supply to the brain, which may be due to abrupt blockage of arteries leading to brain (ischaemic stroke - 85\% of cases), or due to bleeding in brain tissue as a result of ruptured blood vessels (hemorrhagic stroke - $15 \%$ of cases) [1]. According to the World Health Organization, approximately 15 million people worldwide suffer stroke each year. In 2010, stroke was reported to be the fourth largest cause of death in UK after cancer, heart disease and respiratory disease [1]. In addition to accounting for $11.9 \%$ of total deaths worldwide [3], stroke is the largest cause of complex disability in adults. It is responsible for a greater range of disabilities than any other condition. The most common effects of stroke are physical, causing more than half of all stroke survivors to be dependent on others for everyday activities. Post-stroke patients mainly suffer from muscle weakness, paralysis, stiffness or changes in sensation, making it difficult to move their body parts and to perform even basic activities of daily living. Around $80 \%$ of the survivors experience movement problems and up to $38 \%$ may suffer from spasticity.
Stroke has a greater disability impact than any other chronic disease and presents huge economic burden on the healthcare system of a nation. A study by O. Saka et al. [6] quantified the cost of illness of stroke on the UK economy and tried to estimate the direct and indirect costs associated with stroke. The study classified direct costs into formal care costswhich included costs for inpatient care, diagnostic visits, tests, surgical treatments and medical expenses; and informal care costs which included the cost of professional care as well as care provided by family members. Costs resulting from premature death and loss of productivity were used to estimate the indirect costs. The study estimated the cost of stroke care to be around £9 billion pounds a year. Approximately $49 \%$ of this total was the cost of direct formal care, $27 \%$ was the cost of direct informal care and approximately 24\% was indirect cost. Direct costs associated with stroke were approximately $5.5 \%$ of the total UK expenditure on health care.

Rehabilitation for stroke survivors with impaired motor ability aims at either training patients to relearn motor skills that are lost as a result of damage to brain tissue, or to learn new ways of performing activities to circumvent residual disabilities through different types of physical exercises. This involves participation of a large team of experts including physicians, occupational therapists, rehabilitation nurses and informal care from family members, and work is progressing to try and reduce the requirement for highly trained professionals [2].

The cost of rehabilitation includes both formal and informal direct costs, and covers a large portion of the cost of illness for stroke. Thus, to reduce the impact of stroke on the economy and healthcare system, assistive technologies could be used to reduce costly professional intervention in the rehabilitation process. Devices that allow patients to perform regular exercise on their own, and health professionals to monitor patient's performance, without continuous involvement of health experts, could assist the rehabilitation process to a large extent and allow significant monetary savings [4].

Ease of use is also a significant factor [5], with it being estimated that it is likely that if a system is not straightforward to set up or configure, it will not be used for extensive courses of treatment. In addition patients find the repetitive nature of traditional rehabilitation exercises boring, and so motivation for following a rehabilitation program can become difficult.

Therefore the aim of this project was to design an easy-touse rehabilitation system for post-stroke patients that was 
capable of tracking improvements in patient's motor ability by analysing limb movement during rehabilitation exercise. The basic up-down motion of fingers was chosen as the exemplar training exercise and the objective was to design a system capable of tracking improvements in motor ability of patient's hand, with focus on the following features:

- $\quad$ Ease of use: Stiffness in muscles and difficulty in hand movement might make a device that requires complicated setups and contraptions to be worn, inconvenient for rehabilitative use. Hence, the device should have minimum set up requirements

- Motivation for use: Patients may lose interest, and discontinue or become irregular in performing their training when they are left to do it by themselves. Hence, interesting forms of feedback must be provided to keep the user motivated and interested in regular use of the device.

- Performance Tracking: It should allow the patient, as well as health experts, to track the progress being made as the result of regular exercise, and provide feedback to the user on their performance. It needs to maintain a usage history to track performance as well as frequency of use.

\section{SYSTEM DESIGN}

A system comprising of an embedded device and a computer application was designed as a basic framework to analyze finger movements and track progress. Near electricfield based sensing using a Microchip MGC3130 was used in the embedded device as an input sensor to allow non-contact measurement of finger movements. Maximum finger extension, average speed of finger extension and average number of wobbles/jerks per second during finger movement, were chosen as parameters to track improvements in motor ability, and the embedded device was designed to calculate and record these parameters.

To provide feedback to the user, a MIDI interface was included to allow driving a music synthesizer allowing intuitive aural feedback of progress. In addition, a 3 colour LED was included to allow easy visual indication of progress, as the light changed colour relative to finger distance moved. The device was also required to store data on a local SD card and communicate via USB to a host PC, allowing more traditional analysis and visualization of performance eg a scrolling graph.

\section{A. Sensor Operation}

The MGC3130 is a three-dimensional gesture and tracking controller from Microchip and is based on its patented GestIC technology. It includes an integrated signal driver, a frequency adaptive input path for automatic noise suppression and a digital signal processing unit. It was designed to provide a robust 3D input sensing system for user command input and provides gesture and positional data of human hand in real time [7].

However, the focus of the project was to use it as a proximity detector for measuring finger extensions in a rehabilitation system for post-stroke patients. Electric fields are generated around surfaces carrying electrical charge. Application of alternating voltages (AC) creates variation in the electrical charge stored over time and generates a time varying electromagnetic field. A sinusoidal variation of charge with frequency $f$ results in an electromagnetic wave with wavelength

$$
\lambda=c \cdot f
$$

When the wavelength is much larger than the electrode surface, the magnetic component of the waves are zero, resulting in a quasi-static electrical near field that can be used for sensing conductive objects such as the human body. When a person's hand or finger disturbs the field, field lines are drawn to the hand and shunted to ground. This distorts the generated field.

The MGC3130 uses the principle of electrical near-field sensing for advanced proximity detection. It uses frequencies in the range of $100 \mathrm{kHz}$ and generates waves with wavelengths of around $3 \mathrm{~km}$ which are many times larger than the electrodes used. Thus, it generates a quasi-static electrical near field and then uses receiver electrodes to detect E-field variations at different positions. For this it uses a transmit electrode to generate the field and up to 5 receiver electrodes to sample the field. The MGC3130 is then able to detect these changes in effective capacitance due to the presence of foreign bodies which is converted to a change of capacitance of the order of femtoFarads. The MGC3130 then sends this information onto the rest of the system for processing.

\section{SYSTEM ARCHITECTURE}

The system has the basic architecture as shown in figure 1 . The sensor system communicates via SPI to a microcontroller. In this case we use an embed development board based on an NXP LPC1768 ARM M3 SoC [8]. The data is processed and converted into a change of distance measurement, which is then indicated on an LCD, stored in a file, and transmitted to a PC if connected. The data is compared against target data and the MIDI output and the colour of the LED adjusted accordingly as called for by the training program. Local control is provided by a joystick controller and a menu based system on a local LCD.

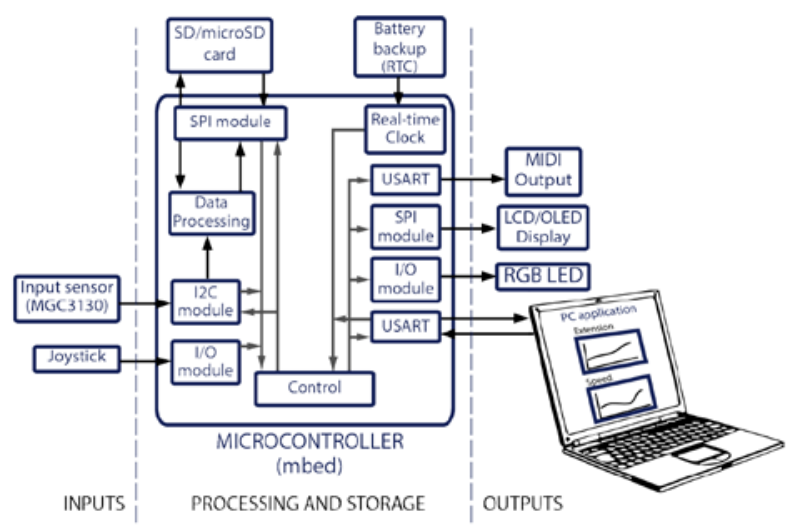

Figure 1: System Architecture 


\section{A. Electrode Design for MGC3130 Controller}

Custom electrodes were designed to improve the sensitivity of the system to the motion of individual fingers. Four electrodes were used and each electrode was placed directly below a finger as shown in Figure 2. The electrodes were arranged to form a bounded region in order to prevent error during the calibration of the Analog Front End (AFE) unit of the MGC3130 controller using the supplied Aurea software. The dimension of the bounded region was kept below $14 \mathrm{~cm} \times 14 \mathrm{~cm}$ as specified in the user guide [9]. The custom electrode system consists of three layers of electrodes separated by polycarbonate sheet with an approximate thickness of $1.4 \mathrm{~mm}$. The top layer consists of four receive (RX) electrodes in the layout shown in Figure 2. The middle layer consists of a rectangular transmit (TX) electrode which is $11.5 \mathrm{~cm} \mathrm{x} 12.5 \mathrm{~cm}$ in dimension; and the bottom layer consists of ground electrode of the same dimension as TX electrode. The width of the widest region of the RX electrodes are approximately $5 \mathrm{~mm}$ and are around $3 \mathrm{~cm}$ long. The narrow region of the RX electrodes are around $2 \mathrm{~mm}$ in width and have been added to define a bounded region. The narrower width was used to decrease the sensitivity of the electrode to the palm and remaining fingers when the hand is close to the electrode (Section 4.3 in [9]). The electrodes were made using shapes cut out of conducting tape (copper).

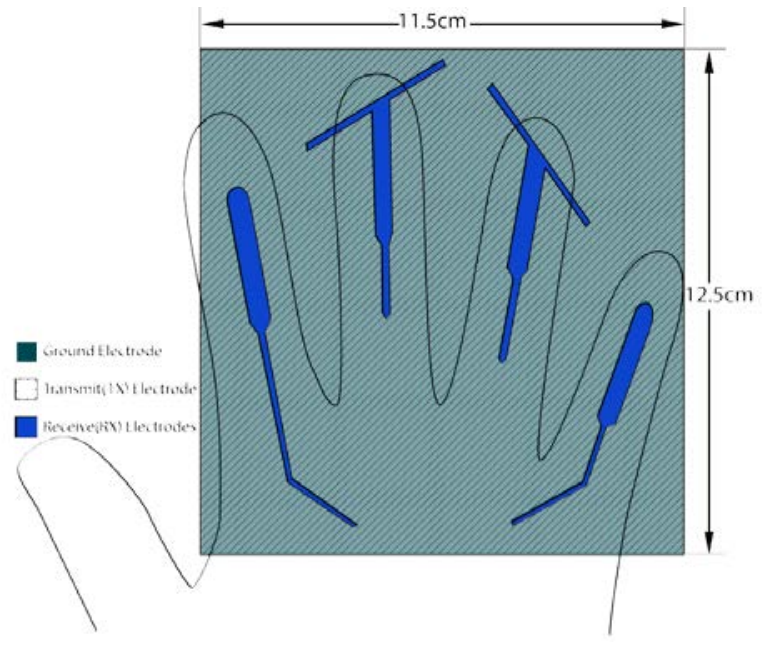

Figure 2: Dimensions and Placement of the electrodes

The equation for plate capacitance,

$$
C=\frac{\varepsilon_{0} \varepsilon_{r} A}{d}
$$

was used for the rough approximation of equivalent capacitances for the electrodes, with $\mathrm{d}=1.4 \mathrm{~mm}$. As suggested in [8], the calculated values were then doubled to include the effect of stray E-field. Table 1 lists the approximate values of equivalent capacitances for different electrodes (doubled to include the effect of stray E-field). The permittivity of polycarbonate (acrylic) sheet $\left(\varepsilon_{\mathrm{r}}\right)$ was taken as 3.5 for calculations. The equivalent capacitances for the designed electrodes are within the limits specified in the electrode design guidelines [9]. $\mathrm{C}_{\mathrm{TxG}}$ (the capacitance of the transmit electrode) is slightly higher than the value recommended for good performance; however it can be decreased by crosshatching of the TX electrode if the electrode system is transferred to a 3-layer PCB.

\begin{tabular}{ccccc}
\hline \multirow{2}{*}{ Electrode } & Approximate & \multicolumn{3}{c}{ Capacitance(pF) } \\
& Area $\left(m^{2}\right)$ & $C_{R x T x}$ & $C_{R x G}$ & $C_{T x G}$ \\
\hline Index & 27 & 1.254 & 0.627 & - \\
Middle & 27 & 1.254 & 0.627 & - \\
Ring & 27 & 1.254 & 0.627 & - \\
Little & 23 & 1.069 & 0.534 & - \\
Transmit(TX) & 14,375 & - & - & 668.20 \\
\hline
\end{tabular}

Table 1:Calculated capacitances

\section{B. Sensor Calibration}

Signal deviation values for each electrode for a graduated increase in distance from the electrode surface were recorded. Figure 4 shows that the data for signal deviation versus distance plot is best approximated by a double exponential curve of the type $y=a e^{b x}+c e^{d x}$.

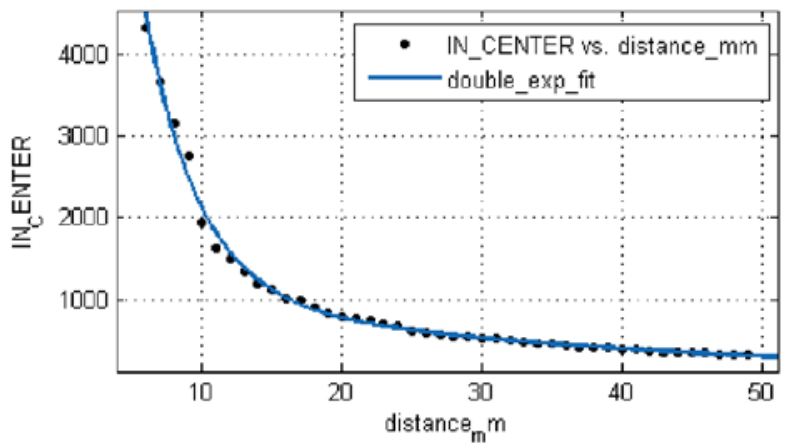

Figure 3: Index finger results showing double exponential fit

Using this curve for distance approximation would require determination of the four parameters (a,b,c,d) for which four exponential equations would have to be solved simultaneously. Also, calculation of distance using this model would involve slower exponential calculations in the microcontroller. The response of the system for different users also varies so that the system needs to calculate these parameters during calibration for each user. To avoid complex calculations, especially during calibration, a simpler piecewise-linear model with four line segments, as given by Equation 3, was used. The parameters for this equation were established by analyzing typical results.

$$
f(x)= \begin{cases}m_{1} x+c_{1}, & \text { if } x \leq 13 m m \\ m_{2} x+c_{2}, & \text { if } 13 m m<x \leq 18 m m \\ m_{3} x+c_{3}, & \text { if } 18 m m<x \leq 23 m m \\ m_{4} x+c_{4}, & \text { otherwise. }\end{cases}
$$




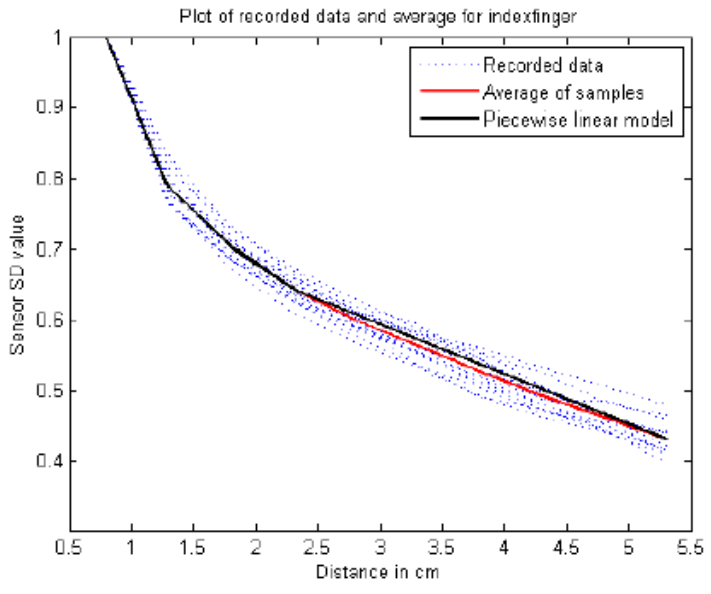

(a) Index finger

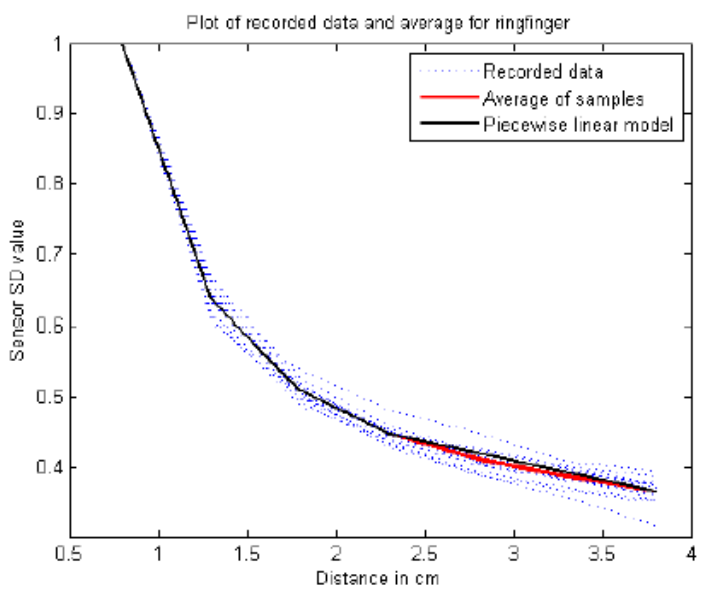

(c) Ring finger

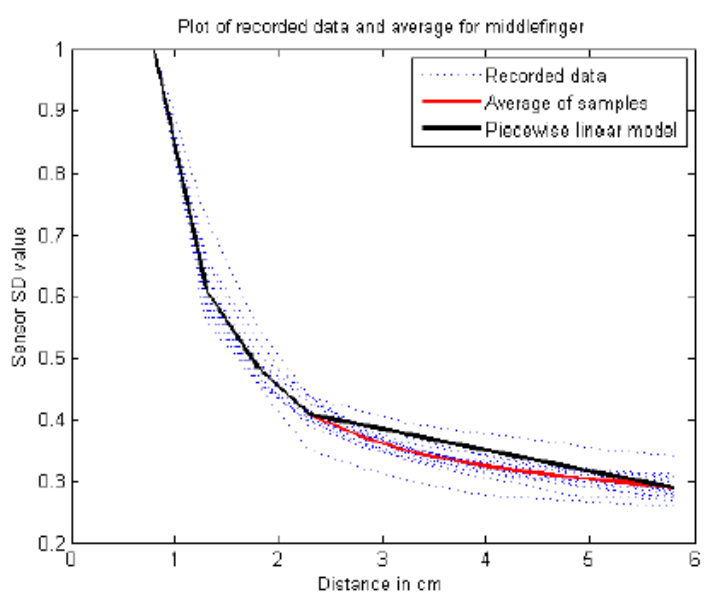

(b) Middle finger

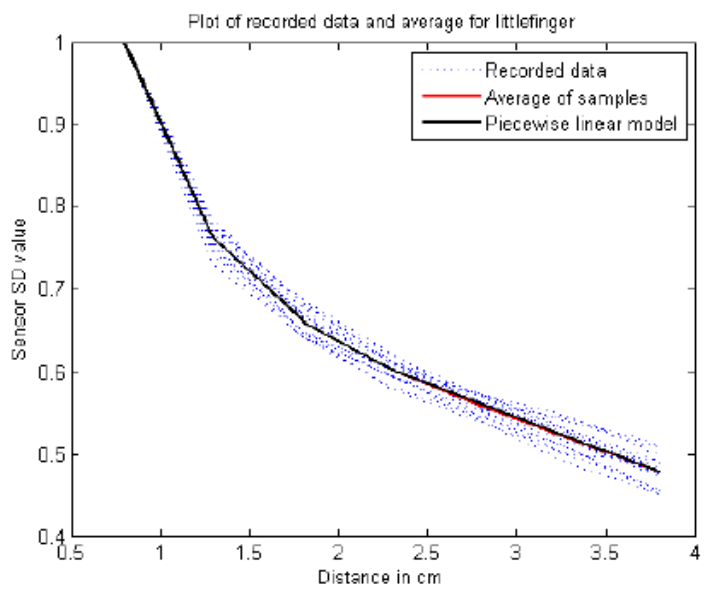

(d) Little finger

Figure 4: Results of 10 trials of raising and lowering each finger

This is illustrated in figure 4, which illustrates how the piecewise model fits real data, in this case consisting of 10 sets of observations. The observations were made by using a ruler to measure the distance moved of the extremity of each finger. Ten sets of observations were made, with each finger being raised and lowered. Figure 4 shows the plots of these observations for all the fingers along with the average for these responses, and the piecewise linear data model obtained for distance calculation. The plots of signal deviation versus distance were normalized against the first signal deviation reading. The slopes $(\mathrm{m} 1 ; \mathrm{m} 2 ; \mathrm{m} 3 ; \mathrm{m} 4)$ and intercepts(c1; c2; c3; c4) of the normalized model, along with normalized signal deviation values of the points of intersection of the four segments, were calculated and stored in configuration files(.cfg) on the SD card and were used for later distance calculation.

\section{Calculation of Extension : Error and Repeatability}

The readings of calculated extensions (not distance from electrode surface but absolute extensions) from the embedded device for four fingers were recorded to observe the error and variability in readings. As mentioned, ten readings for each finger using four extension values were noted. Figure 4 shows that accuracy of model decreases as distance increases; However, as this is targeted at reduced movement cases, we are only really interested in small distances, thus the error was determined for finger extensions of up to $20 \mathrm{~mm}$ with the hand placed $8 \mathrm{~mm}$ above the electrode surface. The error introduced in the readings was largely dependent on the initial placement position of hand; hence, to take the readings, the hand was placed on the device in a position which gave the initial extension reading close to zero millimetres. Table 2 shows the average of the extensions and standard deviations $(\sigma)$ for ten observations recorded for each finger. 


\begin{tabular}{lccccccccc}
\hline & \multicolumn{10}{c}{ Observations } \\
\cline { 2 - 9 } $\begin{array}{l}\text { Ext. } \\
(\mathrm{mm})\end{array}$ & \multicolumn{2}{c}{ Index } & \multicolumn{1}{c}{ Middle } & \multicolumn{2}{c}{ Ring } & \multicolumn{2}{c}{ Little } \\
\cline { 2 - 10 } & Mean & $\sigma(\mathrm{mm})$ & Mean & $\sigma(\mathrm{mm})$ & Mean & $\sigma(\mathrm{mm})$ & Mean & $\sigma(\mathrm{mm})$ \\
\hline 5 & 5.17 & 0.31 & 4.38 & 0.27 & 4.51 & 0.24 & 4.42 & 0.68 \\
10 & 10.48 & 1.18 & 8.48 & 0.34 & 9.11 & 0.31 & 9.35 & 0.28 \\
15 & 16.98 & 0.62 & 13.09 & 0.39 & 14.37 & 0.24 & 14.69 & 0.48 \\
20 & 21.69 & 1.16 & 18.02 & 0.77 & 19.81 & 0.52 & 19.9 & 1.65 \\
\hline
\end{tabular}

Table 2: Standard Deviation and Mean results for extensions up to $20 \mathrm{~mm}$

The results from Table 2 show that extension can be calculated within an error margin of around $2 \mathrm{~mm}$ in a $2 \mathrm{~cm}$ range (actual extension), when the initial position of hand is aligned properly. The variation between readings for repetitions of extensions is also within the margin of two millimeters as indicated by the standard deviation values.

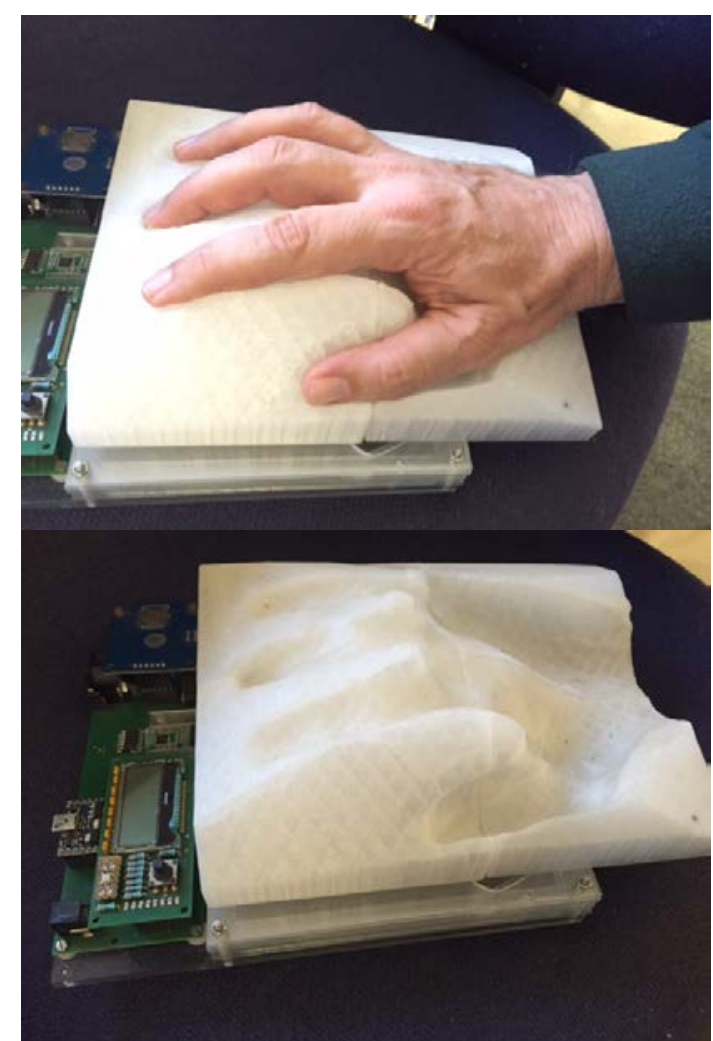

Figure 5: Personalized system with scanned hand receptacle

\section{CONCLUSION AND FUTURE WORK}

The design of an easy-to-use system for use in rehabilitation of post-stroke patients has been discussed and this paper has focused on the design of the distance measuring sensor system. The objective of the project was to design an easy-touse system capable of tracking improvements in motor function of hand as a result of rehabilitation exercise. The designed system, comprising of an embedded system and computer application, meets this objective and provides the basic framework for conveniently tracking improvements in motor ability of hand using MGC3130 gesture and tracking sensor. One of the aims was to emphasize ease of use and this has been demonstrated as once calibrated to the user, the user merely has to place their hand upon the device. It has been established that the best accuracy is achieved when the hand is positioned accurately with respect to the electrodes. Thus future work aims to further improve the ease of use by implementing a personalized system. Figure 5 illustrates this, where a user's hand is scanned and this is used to create a personalized mold. This will greatly assist in accurate placing of the hand, as well as making the device more practical. Although not formally reported, a basic training session (updown finger motion with three repetitions for each finger) was implemented in the system and performance parameters like finger extensions, average response speed and smoothness of finger motion were successfully calculated in real-time. The performance of the sensor was reported, with finger extension measurement calculated with a good accuracy of around $2 \mathrm{~mm}$ within a $2 \mathrm{~cm}$ range using the device. Although not explicitly reported here, features for storing training data for different users into a SD card and comparing performance based on stored data was also successfully implemented. The stored training data could be compared using graphs plotted across timeline to observe progress being made. Output features such as text based feedback, basic music based feedback through a MIDI interface and colour based feedback were also implemented and offer an interesting feedback approach and assessment of these features is continuing, with a view to implementing performance trials.

\section{REFERENCES}

[1] Stroke Association. Stroke statistics, 2013. URL http://www.stroke.org.uk/resource-sheet/stroke-statistics.

[2] Biswas, D., et al. "On the data analysis for classification of elementary upper limb movements." Biomedical Engineering Letters 2014 4(4): 403-413.

[3] World Health Organization. The 10 leading causes of death in the world, 2000 and 2012, http://www.who.int/mediacentre/factsheets/fs310/en/2014.

[4] Burridge, J., Hughes A., "Potential for new technologies in clinical practice” Current Opinion in Neurology 2010 23:6761-677

[5] Hughes, A. et al. "Translation of evidence-based Assistive Technologies into stroke rehabilitation: users' perceptions of the barriers and opportunities” BMC Health Services Research 2014, 14:124

[6] Omer Saka, Alistair McGuire, and CharlesWolfe. Cost of stroke in United Kingdom. Age and Ageing, 2009.

[7] Microchip. Mgc3130 single-zone 3d tracking and gesture controller datasheet,

http://ww1.microchip.com/downloads/en/DeviceDoc/40001667C.pdf.

[8] Mbed LPC1768 https://developer.mbed.org/platforms/mbed-LPC1768/

[9] Microchip. Gestic design guide: Electrodes and system design MGC3130,

http://ww1.microchip.com/downloads/en/DeviceDoc/40001716A.pdf 